\title{
Isolated Avulsion Fracture Lesser Tuberosity of the Humerus, a Rare Presentation Post Seizure
}

\author{
Jaya $T S^{1 *}$, Hadizie $D^{2}$ and TMS Muzaffar ${ }^{3}$ \\ ${ }^{1}$ Medical Officer, Department of Orthopaedic Surgery, Hospital University Sains Malaysia, Malaysia \\ ${ }^{2}$ Lecturer, Department of Orthopaedic Surgeon, Hospital University Sains Malaysia, Malaysia \\ ${ }^{3}$ Associate Professor, Department of Orthopaedic Surgery, Hospital University Sains Malaysia, Malaysia
}

*Corresponding author: Dr. Jaya Thilak, Medical Officer, Department of Orthopaedic Surgery, Hospital University Sains Malaysia, Jalan Raja Perempuan Zainab 2, 16150 Kubang Kerian, Kelantan, Malaysia, Tel: 012-5375037

\begin{abstract}
Isolated humeral lesser tuberosity fracture is rare and is usually associated with fractures of the proximal humerus. Trauma precedes most reported cases. We report a isolated fractured lesser tuberosity occurring in a gentleman post seizure. Open reduction and internal fixation was performed. Functional outcome was successful, and the patient regained his normal pain-free shoulder function 3 months surgery.
\end{abstract}

\section{Keywords}

Isolated lesser tuberosity, Shoulder, Good clinical outcome

\section{Introduction}

Avulsion Fracture Lesser Tuberosity of The Humerus is a rare injury that usually occurs in association with fractures involving two or three parts of the proximal humerus or in association with a posterior fracture dislocation of the shoulder joint [1-3]. The usual mechanism of injury resulting in an avulsion fracture is an abduction external rotation trauma of the shoulder $[2,4,5]$.

Acting as an antagonist, the subscapularis muscle forcefully contracts to resist, leading to an avulsion of the lesser tuberosity [6].

We present a rare case of an isolated lesser tuberosity avulsion fracture post epilepsy that was treated operatively resulting in a good clinical outcome.

\section{Case Report}

A 21-year-old gentleman presented to the Emergency Department with a complaint of left shoulder pain after suffering an epileptic attack a day prior. He could not recall the position of his arm at the time of injury.

Post seizure he experienced left anterior shoulder pain with limited range of motion in his left shoulder. Physical examination revealed an area of ecchymosis and swelling over the anterior left shoulder. Tenderness was localized to the anterior part of the left shoulder. Assessment of his left shoulder showed active forward flexion and abduction was $70^{\circ}$. Active external rotation was normal while active internal rotation was reduced to only $20 \%$. Pain was restricted during active extension and adduction achieved at $15^{\circ}$. The passive range of motion of the left shoulder was $130^{\circ}$ in forward flexion and $130^{\circ}$ in abduction, while the passive extension and adduction was $20^{\circ}$ and $40^{\circ}$, respectively.

Radiograph of the right shoulder taken in the anterior-posterior approach showed a fractured lesser tuberosity found to be displaced caudal to the glenoid (Figure 1). The axillary view clearly revealed an isolated avulsion fracture of lesser tuberosity of the left humerus (Figure 2).

On a later date, a computed tomography scan of the left shoulder was done to assess the fracture configuration, articular extension and its degree of angulation.

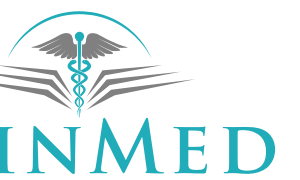

INTERNATIONAL LIBRARY

Citation: Jaya TS, Hadizie D, Muzaffar TMS (2019) Isolated Avulsion Fracture Lesser Tuberosity of the Humerus, a Rare Presentation Post Seizure. Trauma Cases Rev 5:078. doi.org/10.23937/2469$5777 / 1510078$

Accepted: September 16, 2019: Published: September 18, 2019

Copyright: (c) 2019 Jaya TS, et al. This is an open-access article distributed under the terms of the Creative Commons Attribution License, which permits unrestricted use, distribution, and reproduction in any medium, provided the original author and source are credited. 
The avulsed fragment was $19 \mathrm{~mm}$ in length and $14 \mathrm{~mm}$ in width (Figure 3A). The fragment was displaced $8 \mathrm{~mm}$ medially (Figure $3 \mathrm{~B}$ ). The remaining part of the lesser tuberosity was intact and contributed to the medial buttress of the bicipital groove.

Following optimization of his epilepsy, an open reduction and screw fixation of the avulsed fragment was performed. A deltopectoral incision was made to gain access to the fracture fragment. Intraoperatively, the lesser tuberosity was seen to be displaced from its anatomical position, medial to the surgical neck of the humerus. Fracture fragment had no comminution. The subscapularis tendon was found inserted to the posterior portion of the fragment. The avulsed fragment was elevated and realigned to its anatomical location. To secure the tuberosity in its original anatomical position, a single $4.0 \mathrm{~mm}$ compression screw was inserted postero-medially aiming antero-laterally (Figure 4).

Postoperatively, his shoulder was supported in a sling for 2 weeks. Free mobilization of the left shoulder was allowed from the $3^{\text {rd }}$ week onwards once tenderness resolved. Pendulum exercises of the left shoulder was initiated, along with passively assisted range of motion exercises in forward flexion, and external rotation. He progressed to an active range of motion program in the fourth week postoperatively. At the end of the sixth week post-operation, resistive exercises were added. Plain radiographs confirmed the healing of the fracture with good alignment in the $10^{\text {th }}$ week (Figure 5).

On his $3^{\text {rd }}$ month post op follow up, he had regained his normal shoulder function.

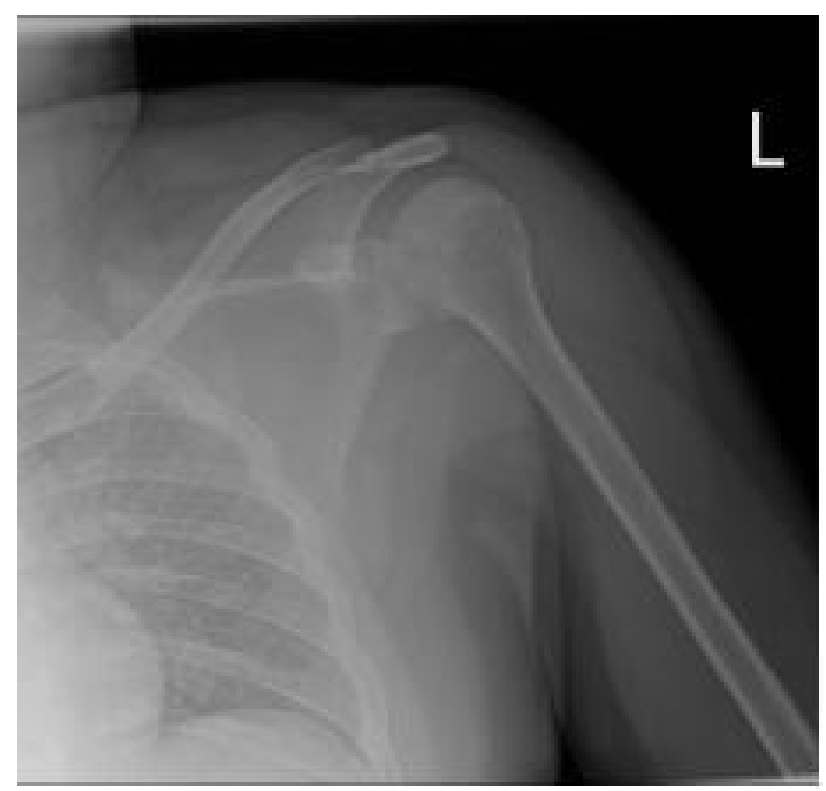

Figure 1: Showed a fractured lesser tuberosity displaced caudal to the glenoid.

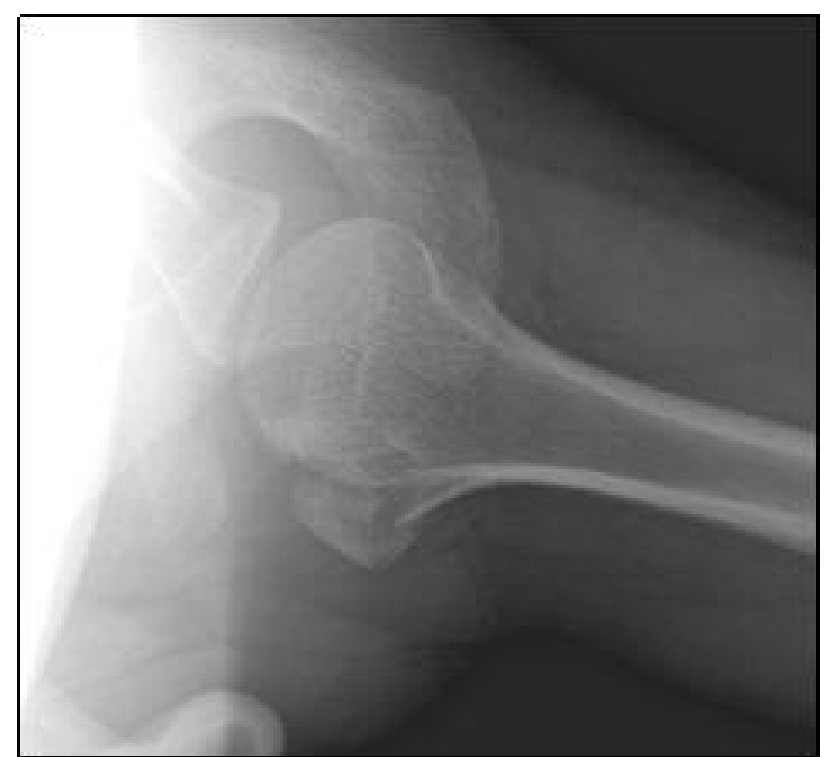

Figure 2: The axillary view of the left shoulder clearly shows the avulsion fragment.

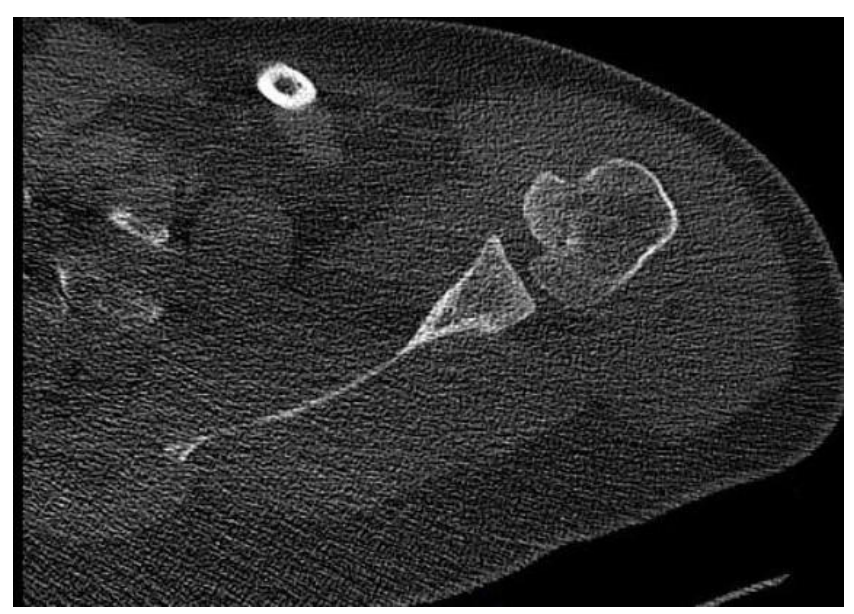

A

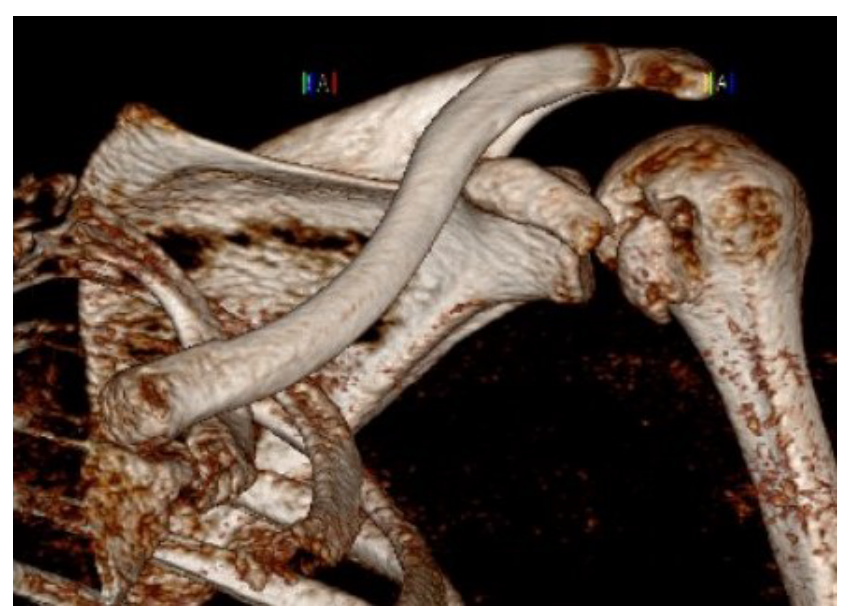

B

Figure 3: A) The axial cut of computer tomography of right shoulder showed the avulsed fragment of lesser tuberosity; B) $3 \mathrm{D}$ reconstruction of computer tomography of left shoulder better defines the size and displacement of fragment with more accurate measurements. 


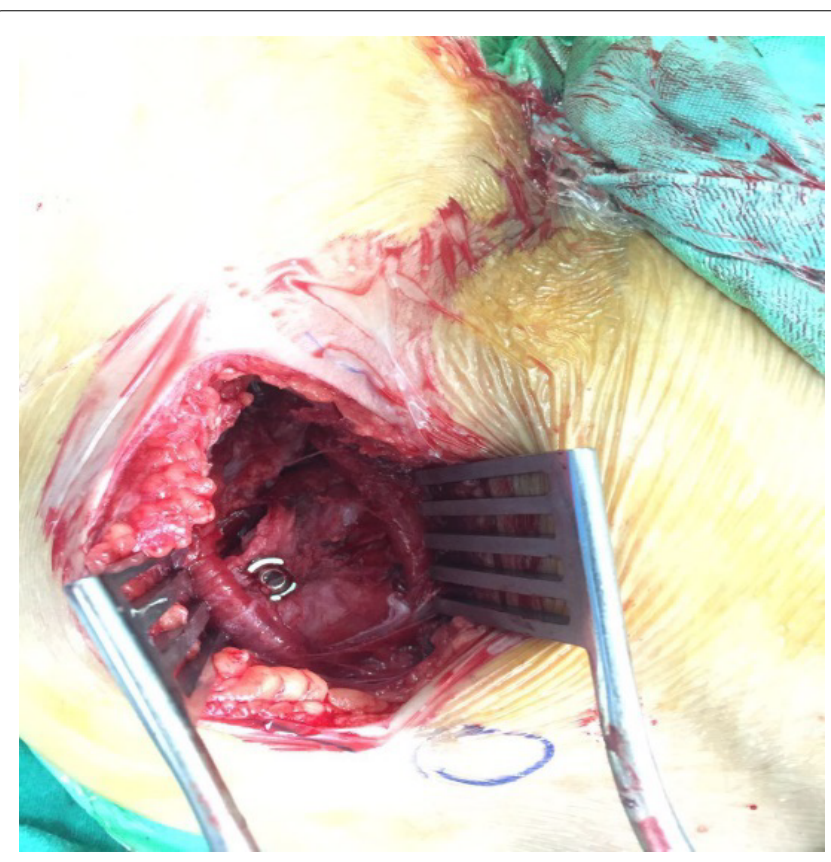

Figure 4: Single compression screw inserted; Intraoperative imaging of fracture fixation using an imaging Intensifier.

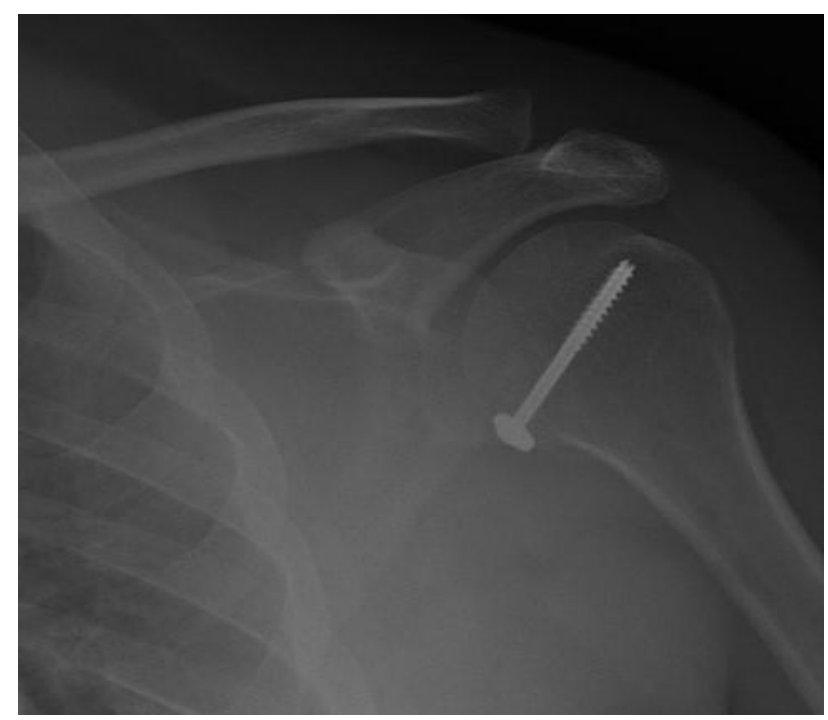

Figure 5: Postoperative anteroposterior radiograph at 10 weeks. Fracture fragment is reduced to its anatomical location with evidence of union.

\section{Discussion}

It is uncommon to have an isolated avulsion fracture of the lesser tuberosity and therefore, experience in the management of this rare injury is less and clinical series remain as few or limited only to case reports $[3,6-9]$. Due to its small size and its location on the medial side of the head of the humerus, the lesser tuberosity is protected from direct injury [3,6-9].

This rare indirect injury is most commonly caused by the pulling forces of the upper gleno-humeral ligament and the subscapularis muscle in hyperextension with increased abduction and external rotation of the shoulder [3].
The main mechanism of injury is believed to be muscular violence where the subscapularis muscle is forced to extend when it forcefully contracts to resist the abduction and external rotation of the shoulder, and this results in a strong traction force which avulses the lesser tuberosity. In keeping with our epileptic patient, involuntary contraction of the subscapularis muscle might have been the cause of the lesser tuberosity fracture [3].

An isolated avulsion fracture of the lesser tuberosity requires a high index of suspicion. Patients presenting with an anterior shoulder tenderness, must be examined thoroughly to look out for tenderness over the lesser tuberosity region. As well as, weakness in performing the 'lift off' test is suggestive of reduced internal rotation and increased passive external rotational, as compared to the contralateral shoulder. Anterior shoulder palpation should be done to suspect subluxation of the long head of biceps tendon and assessment of the gleno-humeral joint stability must be done to rule out associated shoulder dislocations.

With a patient presented with an indirect trauma, an avulsion fracture of the lesser tuberosity can easily be missed with only a routine antero-posterior and scapular view radiographs of the shoulder, especially if the avulsed fragment is minimally displaced. To confirm the diagnosis, an axillary view of the shoulder must be taken [3]. An awareness towards the possibility of an isolated fracture fragment would help in differentiating it from other more common pathologies. A large displaced avulsed fragment can be misdiagnosed to be a bony bankart fragment post shoulder dislocation. Most often an avulsed fragment would be displaced to the sub-glenoid position and this can easily be mistaken as calcified tendonitis [10].

As seen in our case, we suggest a computed tomography scan of the shoulder to be done, to assess articular extension, fracture configuration, size of the fracture fragment and fracture displacement. A clear visualization of the avulsed fragment aids in future fracture management [11].

Ultrasonography and magnetic resonance imaging (MRI) performed for suspected traumatic rotator cuff tear have been shown to be useful in detecting occult tuberosity fractures when initial plain films have been negative. Both modalities may prevent the use of unnecessary diagnostic shoulder arthroscopy for patients with occult fractures [12-14].

Avulsion fractures of the lesser tuberosity is commonly associated with dislocation of the shoulder joint [15].

As mentioned earlier, isolated injuries are rare and easily missed, whereby delayed diagnosis can lead to shoulder instability and impingement symptoms [15].

Shoulder joint instability after an avulsion fracture 
is mainly due to the lengthening of the subscapularis muscle post trauma [16].

In addition to this, a medially displaced avulsed fragment theoretically will narrow the functional space of the coraco-humeral interval, leading to subcoracoid impingement. Although no such as cases have been reported, a subcoracoid impingement present later as chronic shoulder pain [17].

The threshold to perform surgery with regards to the lesser tuberosity displacement in order to maximize patient functional outcome is still controversial. Accepted indication for surgery include fracture fragment displacement of $5 \mathrm{~mm}$ or an angulation of $45^{\circ}$, mechanical blocks to internal rotation, weakness in terminal internal rotation and persistent shoulder pain $[18,19]$.

Possibility of late fracture displacement and involvement of the fracture to the bicipital groove causing potential bicep tendon subluxation has led to some surgeons to fix even minimally displaced fractures. Ogawa, et al. presented one of the largest recent series and recommended ORIF for all acute fractures regardless of fragment size and displacement in order to achieve an excellent long-term outcome [3].

Some authors have recommended non-operative treatment for undisplaced fractures and reported comparable functional outcome to those who underwent surgical intervention [2]. Avulsed fracture fragments have been fixed using cannulated screws $[3,20]$.

As reported in most literatures, open approaches to the humeral lesser tuberosity are limited to the deltopectoral approach. Intraoperatively, most often the subscapularis and avulsed attachment needs to be mobilised to clear adhered fibrous tissues. Keen consideration should be given to the evaluation of the medial wall of the intertubercular groove to preserve the coherence of the tendon of the long head of biceps [15].

Good functional was also obtained by Scheibel, et al. by using an arthroscopic approach with suture anchors for fracture fixation without bicep tendon subluxation [21].

Early post-operative rehabilitation and physiotherapy determine a successful clinical outcome. Rehabilitation of lesser tuberosity fractures follow the similar protocol to rotator cuff injuries. Immediate pendulum exercises are followed by a course of passive range of motion; forward flexion, and internal rotation exercises. Passive motion is advanced to, as tolerated. Early external rotation should be limited to the neutral position in lesser tuberosity repairs.

Active range of motion and stretching exercises are initiated at 6-8 weeks. At 3 months isometric strengthening can be performed $[15,22]$.

\section{Summary}

Isolated avulsion fractures of the lesser tuberosity of the humerus are rare entities, with far lesser experiences reported in comparison to other fractures of the proximal humerus. High index of suspicion when handling cases with anterior shoulder pain could safe a patient from a poorly functioning shoulder. Axillary view radiograph and computed tomography scan will aid diagnosis. As in other fracture fixations, a successful functional outcome of humeral lesser tuberosity fracture relies strongly on the right indication to operate, good surgical techniques and a compliant rehabilitation regime.

\section{References}

1. Levine B, Pereira D, Rosen J (2005) Avulsion fractures of the lesser tuberosity of the humerus in adolescents: Review of the literature and case report. J Orthop Trauma 19: 349352.

2. Van Laarhoven HA, te Slaa RL, van Laarhoven EW (1995) Isolated avulsion fracture of the lesser tuberosity of the humerus. J Trauma 39: 997-999.

3. Ogawa K, Takahashi M (1997) Long-term outcome of isolated lesser tuberosity fractures of the humerus. J Trauma 42: 955-959.

4. Ross GJ, Love MB (1989) Isolated avulsion fracture of the lesser tuberosity of the humerus: Report of two cases. Radiology 172: 833-834.

5. Pace A, Ribbans W, Kim JH (2008) Isolated lesser tuberosity fracture of the humerus. Orthopedics 31: 94.

6. Kanso I, Bricout JM (1998) Isolated avulsion fracture of the lesser tuberosity of the humerus. Apropos of a case. Rev Chir Orthop Reparatrice Appar Mot 84: 554-557.

7. Caniggia M, Maniscalco P, Picinotti A (1996) Isolated avulsion fracture of the lesser tuberosity of the humerus. Report of two cases. Panminerva Med 38: 56-60.

8. Berbig R, Keller H, Metzger U (1994) Isolated fracture of the lesser tuberosity of the humerus: Case reports and review of the literature. Z Unfallchir Versicherungsmed 87: 159-168.

9. Thielemann FW, Kley U, Holz U (1992) Isolated injury of the subscapular muscle tendon. Sportverletz Sportschaden 6: 26-28.

10. Shibuya S, Ogawa K (1986) Isolated avulsion fracture of the lesser tuberosity of the humerus. A case report. Clin Orthop Relat Res 211: 215-218.

11. Mora Guix JM, Gonzalez AS, Brugalla JV, Carril EC, Baños FG (2006) Proposed protocol for reading images of humeral head fractures. Clin Orthop Relat Res 448: 225-233.

12. Mason BJ, Kier R, Bindleglass DF (1999) Occult fractures of the greater tuberosity of the humerus: Radiographic and MR imaging findings. AJR Am J Roentgenol 172: 469-473.

13. Patten RM, Mack LA, Wang KY, Lingel J (1992) Nondisplaced fractures of the greater tuberosity of the humerus: Sonographic detection. Radiology 182: 201-204.

14. Reinus WR, Hatem SF (1998) Fractures of the greater tuberosity presenting as rotator cuff abnormality: Magnetic resonance demonstration. J Trauma 44: 670-675.

15. Gruson KI, Ruchelsman DE, Tejwani NC (2008) Isolated tuberosity fractures of the proximal humerus: Current concepts. Injury 39: 284-298. 
16. Symeonides PP (1972) The significance of the subscapularis muscle in the pathogenesis of recurrent anterior dislocation of the shoulder. J Bone Joint Surg Br 54: 476-483.

17. Hung LH, Chung KY, Tang N, Leung KS (2012) Isolated avulsion fracture of the lesser tuberosity of the humerus: Case report and literature review. Journal of Orthopaedics, Trauma and Rehabilitation 16: 78-81.

18. Park TS, Choi IY, Kim YH, Park MR, Shon JH, et al. (1997) A new suggestion for the treatment of minimally displaced fractures of the greater tuberosity of the proximal humerus. Bull Hosp Joint Dis 56: 171-176.

19. Paschal SO, Hutton KS, Weatherall PT (1995) Isolated avulsion fracture of the lesser tuberosity of the humerus in adolescents. A report of two cases. J Bone Joint Surg Am 77: 1427-1430.

20. Hayes PR, Klepps S, Bishop J, Cleeman E, Flatow EL (2003) Posterior shoulder dislocation with lesser tuberosity and scapular spine fractures. J Shoulder Elbow Surg 12: 524-527.

21. Scheibel M, Martinek V, Imhoff AB (2005) Arthroscopic reconstruction of an isolated avulsion fracture of the lesser tuberosity. Arthroscopy 21: 487-494.

22. Koval KJ, Gallagher MA, Marsicano JG, Cuomo F, McShinawy A, et al. (1997) Functional outcome after minimally displaced fractures of the proximal part of the humerus. $J$ Bone Joint Surg Am 79: 203-207. 